\title{
A DISTRIBUTION ON TRIPLES WITH MAXIMUM ENTROPY MARGINAL
}

\author{
SERGEY NORIN \\ Department of Mathematics and Statistics, McGill University, Montréal, Canada; \\ email: sergey.norin@mcgill.ca
}

Received 9 January 2017; accepted 17 November 2019

\begin{abstract}
We construct an $S_{3}$-symmetric probability distribution on $\left\{(a, b, c) \in \mathbb{Z}_{\geqslant 0}^{3}: a+b+c=n\right\}$ such that its marginal achieves the maximum entropy among all probability distributions on $\{0,1, \ldots, n\}$ with mean $n / 3$. Existence of such a distribution verifies a conjecture of Kleinberg et al. ['The growth rate of tri-colored sum-free sets', Discrete Anal. (2018), Paper No. 12, arXiv:1607.00047v1], which is motivated by the study of sum-free sets.
\end{abstract}

2010 Mathematics Subject Classification: 11B30 (primary); 05D40 (secondary)

\section{Introduction}

The recent breakthrough by Croot, Lev and Pach [2] and the subsequent solution of the cap-set problem by Ellenberg and Gijswijt [3] led to a dramatic improvement of known upper bounds on the size of maximum sum-free sets in powers of finite groups. Blasiak et al. [1] extended the Ellenberg-Gijswijt result to multicolored sum-free sets. Even more recently, Kleinberg, Sawin and Speyer [5] established upper bounds for the multicolored version which are essentially tight. Let us state the main result of [5], which motivates our work.

Let $p$ be a prime. A tri-colored sum-free set in $\mathbb{F}_{p}^{n}$ is a collection of triples $\left\{\left(x_{i}\right.\right.$, $\left.\left.y_{i}, z_{i}\right)\right\}_{i=1}^{m}$ of elements of $\mathbb{F}_{p}^{n}$ such that $x_{i}+y_{j}+z_{k}=0$ if and only if $i=j=k$. Kleinberg, Sawin and Speyer establish an upper bound $m \leqslant e^{\gamma_{p} n}$ on the size of a tri-colored sum-free set in $\mathbb{F}_{p}^{n}$, where $\gamma_{p}$ is as follows.

The entropy of a probability distribution $\mu$ on a finite set $I$ is defined as

$$
\eta(\mu)=\sum_{i \in I} \mu(i) \log \mu(i)
$$

(c) The Author 2019. This is an Open Access article, distributed under the terms of the Creative Commons Attribution licence (http://creativecommons.org/licenses/by/4.0/), which permits unrestricted re-use, distribution, and reproduction in any medium, provided the original work is properly cited. 
where we interpret $0 \log 0$ as 0 . Let $T=\left\{(a, b, c) \in \mathbb{Z}_{\geqslant 0}^{3}: a+b+c=p-\right.$ 1 . Let $\pi$ be an $S_{3}$-symmetric probability distribution on $T$, and let $\mu(\pi)$ be the marginal probability distribution of $\pi$ on $[0, p-1]$ corresponding to the first coordinate. (We denote the set of consecutive integers $\{n, n+1, \ldots, n+k\}$ by $[n, n+k]$.) (As $\pi$ is $S_{3}$-symmetric, the choice of a coordinate is irrelevant.) Let $\gamma_{p}$ be the maximum entropy of $\mu(\pi)$ over $S_{3}$-symmetric probability distributions $\pi$ on $T$.

THEOREM 1 (Kleinberg, Sawin and Speyer [5]). (In the published version of [5], the upper bound part of the statement of Theorem 1, as well as the examples for $n \leqslant 25$ referenced later, were removed, as the proofs of Theorem 2 in an earlier version of this article, and independent work of Pebody [6], showed that they were unnecessary, but they are still available in the referenced arxiv version.) All tri-colored sum-free sets in $\mathbb{F}_{p}^{n}$ have size at most $e^{\gamma_{p}{ }^{n}}$. Moreover, there exist tri-colored sum-free sets in $\mathbb{F}_{p}^{n}$ of size at least $e^{\gamma_{p} n-o(n)}$.

Every marginal of a symmetric probability distribution on $T$ has mean $(p-1) / 3$. Therefore, $\gamma_{p}$ is at most the maximum entropy of a probability distribution on $[0, p-1]$ with this mean. The main result of this paper, which was independently obtained by Pebody [6], gives a proof of [5, Theorem 4] showing that the equality holds.

THEOREM 2. For every $n \geqslant 1$, there exists an $S_{3}$-symmetric probability distribution $\pi$ on $\left\{(a, b, c) \in \mathbb{Z}_{\geqslant 0}^{3}: a+b+c=n\right\}$ such that $\mu(\pi)$ achieves the maximum entropy among probability distributions on $[0, n]$ with mean $n / 3$.

While the definition of $\gamma_{p}$ above already implies that it is a computable constant, Theorem 2 provides a much simpler description. As noted in [5], a direct calculation shows that if $\mu$ has the maximum entropy among probability distributions on $[0, n]$ with mean $n / 3$, then

$$
\mu(i)=\frac{\rho^{i}}{1+\rho+\cdots+\rho^{n}},
$$

where $\rho$ is the unique positive real solution to the equation

$$
\sum_{i=0}^{n} i \rho^{i}=\frac{n}{3} \sum_{i=0}^{n} \rho^{i} .
$$

Further, Theorem 2 confirms that the upper bound in Theorem 1 coincides with the bounds established for sum-free sets in [3] and three-colored sum-free sets 
in [1]. The value of $\gamma_{p}$ is also of interest as it appears in the tight bound for the arithmetic triangle removal lemma of Fox and Lovász [4].

We construct a distribution $\pi$ satisfying Theorem 2 explicitly. Examples of the distributions satisfying Theorem 2 for $n \leqslant 25$ are provided in [5]. Based on these examples and additional experimentation, we construct a simple $S_{3}$-symmetric function of $\left\{(a, b, c) \in \mathbb{Z}_{\geqslant 0}^{3}: a+b+c=n\right\}$ with the marginal given by (1). This construction is presented in Section 2 along with the necessary notation. Unfortunately, the constructed function fails to be nonnegative for $n \geqslant 28$. In Section 3, we modify via a sequence of 'local' changes to establish Theorem 2 in full generality.

\section{Notation and the first attempt}

Fix positive integer $n$ for the remainder of the paper. Let $T=\left\{(a, b, c) \in \mathbb{Z}_{\geqslant 0}^{3}\right.$ : $a+b+c=n\}$. The probabilistic distributions we are interested in form a polytope in $\mathbb{R}^{T}$, and vectors in $\mathbb{R}^{T}$ will be the main object of study in the remainder of the paper. We use the convention $\boldsymbol{v}=\left(v_{a b c}\right)_{(a, b, c) \in T}$, that is, we denote by $v_{a b c}$ the component of the vector $v$ corresponding to a triple $(a, b, c) \in T$. Let $\{\boldsymbol{e}(a, b$, $c)\}_{(a, b, c) \in T}$ be the standard basis of $\mathbb{R}^{T}$. We say that a vector $\boldsymbol{v} \in \mathbb{R}^{T}$ is symmetric if $v_{i_{1} i_{2} i_{3}}=v_{i_{\sigma(1)} i_{\sigma(2)} i_{\sigma(3)}}$ for every permutation $\sigma \in S_{3}$. Let $W \subseteq \mathbb{R}^{T}$ be the vector space of symmetric vectors. For $\left(i_{1}, i_{2}, i_{3}\right) \in T$, define

$$
\boldsymbol{s}\left(i_{1}, i_{2}, i_{3}\right)=\sum_{\sigma \in S_{3}} \boldsymbol{e}\left(i_{\sigma(1)}, i_{\sigma(2)}, i_{\sigma(3)}\right)
$$

The set $\{s(a, b, c) \mid(a, b, c) \in T, a \leqslant b \leqslant c\}$ forms a convenient basis of $W$. For $\boldsymbol{v} \in \mathbb{R}^{T}$ and $a \in[0, n]$, define

$$
\mu_{a}(\boldsymbol{v})=\sum_{i=0}^{n-a} v_{a i(n-a-i)},
$$

and let $\mu: \mathbb{R}^{T} \rightarrow \mathbb{R}^{[0, n]}$ be defined by

$$
\mu(\boldsymbol{v})=\left(\mu_{0}(\boldsymbol{v}), \mu_{1}(\boldsymbol{v}), \ldots, \mu_{n}(\boldsymbol{v})\right) .
$$

Note that importantly

$$
\mu_{i}(s(a, b, c))=2\left(\delta_{i a}+\delta_{i b}+\delta_{i c}\right),
$$

for $i \in[0, n]$ and $(a, b, c) \in T$, where $\delta$ is the Kronecker delta.

Let $\rho$ de defined by (2). Clearly, $\rho<1$. Define

$$
\boldsymbol{r}=\left(n, n \rho, \ldots, n \rho^{n}\right) \in \mathbb{R}^{[0, n]} .
$$


We say that $\boldsymbol{v} \in \mathbb{R}^{T}$ is $\rho$-marginal if $\mu(\boldsymbol{v})=\boldsymbol{r}$. Let $R$ denote the set of symmetric, $\rho$-marginal vectors in $\mathbb{R}^{T}$. Note that $R$ is an affine space and $R=\boldsymbol{v}+(\operatorname{Ker}(\mu) \cap W)$ for any $v \in R$.

It is easy to see that the next theorem is a reformulation of Theorem 3 using the introduced terminology. (Note that, for convenience, we scaled the target marginal by $n\left(1+\rho+\cdots+\rho^{n}\right)$.)

THEOREM 3. There exists a nonnegative vector $\pi \in R$.

We construct an explicit, albeit not particularly elegant, vector $\pi$ satisfying Theorem 3. As a first step in this section, we construct an auxiliary vector $\beta \in R$, which has a compact description and will be the starting point of the general construction. In Section 3, we finish the construction of $\pi$ by defining a generating set of $\operatorname{Ker}(\mu)$ consisting of vectors with small support and adding an appropriate linear combination of these vectors to $\boldsymbol{\beta}$.

We now define $\boldsymbol{\beta}$. Let

$$
\beta_{a b c}=\rho^{a}-\rho^{n-a}+\rho^{b}-\rho^{n-b}+\rho^{c}-\rho^{n-c},
$$

for $(a, b, c) \in T, a, b, c \geqslant 1$, let

$$
\beta_{a 0(n-a)}=\sum_{i=1}^{a-1}\left(-\rho^{i}+\rho^{n-i}\right)+\frac{a-1}{2} \rho^{a}+\frac{n-a+1}{2} \rho^{n-a},
$$

for $1 \leqslant a \leqslant n / 2$, let

$$
\beta_{00 n}=n \rho^{n}
$$

and define the components of $\boldsymbol{\beta}$ for the remaining triples in $T$ by symmetry so that $\boldsymbol{\beta}$ is symmetric.

LEMMA 4. The vector $\boldsymbol{\beta}$ is $\rho$-marginal.

Proof. First, let us note that the identity (5) also holds for $n / 2<a \leqslant n-1$. Indeed, for such $i$, we have

$$
\begin{aligned}
\beta_{a 0(n-a)} & =\sum_{i=1}^{n-a-1}\left(-\rho^{i}+\rho^{n-i}\right)+\frac{n-a-1}{2} \rho^{n-a}+\frac{a+1}{2} \rho^{a} \\
& =\sum_{i=1}^{n-a-1}\left(-\rho^{i}+\rho^{n-i}\right)+\sum_{i=n-a}^{a}\left(-\rho^{i}+\rho^{n-i}\right)+\frac{n-a-1}{2} \rho^{n-a}+\frac{a+1}{2} \rho^{a} \\
& =\sum_{i=1}^{a-1}\left(-\rho^{i}+\rho^{n-i}\right)-\rho^{a}+\rho^{n-a}+\frac{n-a-1}{2} \rho^{n-a}+\frac{a+1}{2} \rho^{a}
\end{aligned}
$$




$$
=\sum_{i=1}^{a-1}\left(-\rho^{i}+\rho^{n-i}\right)+\frac{a-1}{2} \rho^{a}+\frac{n-a+1}{2} \rho^{n-a},
$$

as desired.

Now we are ready to verify that $\mu_{a}(\boldsymbol{\beta})=n \rho^{a}$ for every $a \in[0, n]$. We have $\mu_{n}(\boldsymbol{\beta})=n \rho^{n}$ by $(6)$. For $1 \leqslant a \leqslant n-1$, we have

$$
\begin{aligned}
\mu_{a}(\boldsymbol{\beta})= & \sum_{i=0}^{n-a} \beta_{a i(n-a-i)}=\sum_{i=1}^{n-a-1}\left(\rho^{a}-\rho^{n-a}+\rho^{i}-\rho^{n-i}+\rho^{n-a-i}-\rho^{a+i}\right) \\
& +2 \sum_{i=1}^{a-1}\left(-\rho^{i}+\rho^{n-i}\right)+(a-1) \rho^{a}+(n-a+1) \rho^{n-a} \\
= & (n-a-1)\left(\rho^{a}-\rho^{n-a}\right)+2 \sum_{i=1}^{n-a-1}\left(\rho^{i}-\rho^{n-i}\right)-2 \sum_{i=1}^{a-1}\left(\rho^{i}-\rho^{n-i}\right) \\
& +(a-1) \rho^{a}+(n-a+1) \rho^{n-a} \\
= & (n-2) \rho^{a}+2 \rho^{n-a}+2\left(\rho^{a}-\rho^{n-a}\right)=n \rho^{a},
\end{aligned}
$$

as desired. Finally, we have

$$
\begin{aligned}
\mu_{0}(\boldsymbol{\beta}) & =\sum_{i=0}^{n} \beta_{0 i(n-i)} \\
& =2 n \rho^{n}+\sum_{i=1}^{n-1}\left(\sum_{j=1}^{i-1}\left(-\rho^{j}+\rho^{n-j}\right)+\frac{i-1}{2} \rho^{i}+\frac{n-i+1}{2} \rho^{n-i}\right) \\
& =2 n \rho^{n}+\sum_{i=1}^{n-1}(-(n-i-1)+(i-1)+i) \rho^{i} \\
& =\sum_{i=1}^{n}(3 i-n) \rho^{i}=n+\sum_{i=0}^{n}(3 i-n) \rho^{i}=n
\end{aligned}
$$

where the last equality uses (2).

Upon cursory examination, $\boldsymbol{\beta}$ appears to be a promising candidate for a nonnegative vector in $R$. In fact, $\beta$ is the only vector in $R$ for $n \leqslant 5$. In general, it is easy to see that $\beta_{a b c} \geqslant 0$ for $a, b, c \geqslant 1$. Unfortunately, $\boldsymbol{\beta}$ is nonnegative only for $n \leqslant 27$, whereas $\beta_{0\lfloor n / 2\rfloor\lceil n / 2\rceil}<0$ for $n \geqslant 28$. Thus, we have to modify $\boldsymbol{\beta}$ by adding to it an appropriate vector in $\operatorname{Ker}(\mu)$. This is the goal of the next, somewhat technical section. 


\section{Flattening $\beta$}

Given $(a, b, c) \in T$ and $x, y \in \mathbb{N}$ such that $b \geqslant x, c \geqslant x+y$, we define a vector

$$
\begin{aligned}
\boldsymbol{m}^{x, y}(a, b, c)= & -\boldsymbol{s}(a, b, c)+\boldsymbol{s}(a+x, b-x, c) \\
& -\boldsymbol{s}(a+x+y, b-x, c-y)+\boldsymbol{s}(a+x+y, b, c-x-y) \\
& -\boldsymbol{s}(a+x, b+y, c-x-y)+\boldsymbol{s}(a, b+y, c-y) .
\end{aligned}
$$

Claim 5. We have $\boldsymbol{m}^{x, y}(a, b, c) \in \operatorname{Ker}(\mu) \cap W$ for all $(a, b, c) \in T$ and $x, y \in \mathbb{N}$ such that $b \geqslant x, c \geqslant x+y$.

Proof. Clearly, $\boldsymbol{m}^{x, y}(a, b, c) \in W$. Therefore, we only need to show that $\mu_{i}\left(\boldsymbol{m}^{x, y}(a, b, c)\right)=0$ for every $i \in[0, n]$. This follows immediately from (3), as each Kronecker deltas $\delta_{i j}$ for $j \in\{a, a+x, a+x+y, b, b-x, b+y, c, c-y$, $c-x-y\}$ will appear the same number of times with positive and negative signs in the expansion of $\mu_{i}\left(\boldsymbol{m}^{x, y}(a, b, c)\right)$ using (3).

We think of vectors $\boldsymbol{m}^{x, y}(a, b, c)$ as directions, along which a vector in $R$ can be shifted to obtain another vector in $R$ differing from the original only in a few coordinates. In the remainder of the section, we describe a collection of such shifts which transform $\boldsymbol{\beta}$ into a nonnegative vector.

We will only use a subset of the vectors defined above of the following form. $\boldsymbol{m}(b \rightarrow a)=\boldsymbol{m}^{\lceil b / 2\rceil, a-b}(0, b, n-b)$ for $2 \leqslant b<a \leqslant n / 2$. That is,

$$
\begin{aligned}
\boldsymbol{m}(b \rightarrow a)= & -s(0, b, n-b)+\boldsymbol{s}(\lceil b / 2\rceil,\lfloor b / 2\rfloor, n-b) \\
& -s(a-\lfloor b / 2\rfloor,\lfloor b / 2\rfloor, n-a)+s(a-\lfloor b / 2\rfloor, b, n-a-\lceil b / 2\rceil) \\
& -s(\lceil b / 2\rceil, a, n-a-\lceil b / 2\rceil)+s(0, a, n-a) .
\end{aligned}
$$

We obtain $\pi$ from $\boldsymbol{\beta}$ by adding to it a linear combination of vectors $\boldsymbol{m}(b \rightarrow a)$. The coefficients of these vectors, which we define next, are chosen so that, in particular, $\pi_{0 a(n-a)}=\pi_{0 b(n-b)}$ for $2 \leqslant a, b \leqslant n-2$. (Hence, we think of the construction of $\boldsymbol{\pi}$ as 'flattening $\boldsymbol{\beta}$ '.) Denote $\beta_{0 i(n-i)}$ by $z_{i}$ for brevity, and let

$$
\begin{aligned}
c_{b} & =\frac{2}{n+1-2 b}\left(z_{b}-\frac{1}{n-1-2 b} \sum_{i=b+1}^{n-b-1} z_{i}\right) \\
& =\frac{\sum_{i=b}^{n-b} z_{i}}{n+1-2 b}-\frac{\sum_{i=b+1}^{n-b-1} z_{i}}{n-1-2 b} .
\end{aligned}
$$

Let $c_{b a}=c_{b}$ for $2 \leqslant b<a<n / 2$ and let $c_{b(n / 2)}=\frac{c_{b}}{2}$ for even $n$. 
We are now ready to define $\pi$ :

$$
\boldsymbol{\pi}=\boldsymbol{\beta}+\sum_{2 \leqslant b \leqslant n / 2-1} \sum_{b+1 \leqslant a \leqslant n / 2} c_{b a} \boldsymbol{m}(b \rightarrow a)
$$

In the remainder of the section, we show that $\pi$ satisfies Theorem 3. It follows from Claim 5 that $\pi \in R$. Thus, it remains to show that $\pi_{a b c} \geqslant 0$ for $(a, b, c) \in T$. This is accomplished in the following series of claims.

Claim 6. For $2 \leqslant a \leqslant n / 2$, we have

$$
\pi_{0 a(n-a)}=\frac{n}{n-2}\left(1-\rho^{n}-\rho^{n-1} / 2\right) \geqslant 0 .
$$

Proof. We have $\pi_{00 n}=n \rho^{n}, \pi_{01 n}=n \rho^{n-1} / 2$ and $\mu_{0}(\pi)=n$, as $\pi$ is $\rho$-marginal. Thus, to establish (9), it suffices to verify that $\pi_{0 a n-a}=\pi_{0 b n-b}$ for $2 \leqslant a, b \leqslant n-2$. Define

$$
\boldsymbol{\pi}^{i}=\boldsymbol{\beta}+\sum_{i \leqslant b \leqslant n / 2-1} \sum_{b+1 \leqslant a \leqslant n / 2} c_{b a} \boldsymbol{m}(b \rightarrow a) .
$$

We will show by induction on $n / 2-b$ that

$$
\pi_{0 a(n-a)}^{b}=\frac{\sum_{i=b}^{n-b} z_{i}}{n+1-2 b}
$$

for every $b \leqslant a \leqslant n-b$. The identity (10) for $b=2$ implies the claim.

The base case $b=\lfloor n / 2\rfloor$ is trivial. For the induction step and $b<a<n-b$

$$
\begin{aligned}
\pi_{0 a(n-a)}^{b} & =\pi_{0 a(n-a)}^{b+1}+c_{b} \\
& =\frac{\sum_{i=b}^{n-b} z_{i}}{n-1-2 b}+\left(\frac{\sum_{i=b}^{n-b} z_{i}}{n+1-2 b}-\frac{\sum_{i=b+1}^{n-b-1} z_{i}}{n-1-2 b}\right) \\
& =\frac{\sum_{i=b}^{n-b} z_{i}}{n+1-2 b},
\end{aligned}
$$

as desired, as $z_{b}=z_{n-b}$. Finally,

$$
\begin{aligned}
\pi_{0 b(n-b)}^{b} & =\pi_{0 b(n-b)}^{b+1}-\frac{n-1-2 b}{2} c_{b} \\
& =z_{b}-\frac{n-1-2 b}{n+1-2 b}\left(z_{b}-\frac{1}{n-1-2 b} \sum_{i=b+1}^{n-b-1} z_{i}\right) \\
& =\frac{\sum_{i=b}^{n-b} z_{i}}{n+1-2 b},
\end{aligned}
$$

finishing the proof of the identity in (10). 
It remains to show that $\rho^{n}+\rho^{n-1} / 2 \leqslant 1$. Using (2), we have

$$
\begin{aligned}
\frac{n(n+1)}{3}\left(\rho^{n}+\frac{\rho^{n-1}}{2}\right) & \leqslant n \rho^{n}+\frac{n(n-1)}{2} \rho^{n-1} \\
& \leqslant \sum_{i=0}^{n} i \rho^{i}=\frac{n}{3} \sum_{i=0}^{n} \rho^{i} \leqslant \frac{n(n+1)}{3},
\end{aligned}
$$

implying the desired inequality.

We now proceed to establish the estimates which will allow us to prove the nonnegativity of $\pi$. We start with a couple of indirect bounds on $\rho$.

Claim 7. We have

$$
\rho^{n / 2+1} \geqslant \frac{2}{3 e}
$$

Proof. It can be verified by a computer that $\rho^{n / 2+1} \geqslant 1 / 3$ for $n \leqslant 15$. Thus, (11) holds for $n \leqslant 15$, and we assume $n \geqslant 16$.

Let $\alpha=(n+2)(1-\rho) / \rho$. Then $\rho=(n+2) /(n+2+\alpha)$, and

$$
\rho^{n+1} \geqslant \rho^{n+2}=\frac{1}{(1+\alpha /(n+2))^{n+2}} \geqslant e^{-\alpha} .
$$

We claim that $\alpha \leqslant 2 \log (3 e / 2)$. Note that by (12), this claim implies (11).

We start the proof of the claim by multiplying both sides of (2) by $(1-\rho)^{2}$, expanding and rearranging terms to obtain

$$
(n+3) \rho-n-\rho^{n+1}((2 n+3)-2 n \rho)=0 .
$$

Using (12) to bound $\rho^{n+1}$ and otherwise expressing $\rho$ in terms of $\alpha$, we get

$$
\frac{(n+3)(n+2)}{n+2+\alpha}-n-e^{-\alpha}\left(2 n+3-\frac{2 n(n+2)}{n+2+\alpha}\right) \geqslant 0 .
$$

Multiplying the above inequality by $n+2+\alpha$, we obtain

$$
3 n+6-n \alpha-e^{-\alpha}(3 n+6+(2 n+3) \alpha) \geqslant 0 .
$$

Let $f(x, n)=3 n+6-n x-e^{-x}(3 n+6+(2 n+3) x)$. We have $f(2 \log (3 e / 2)$, $16)=-0.14055 \ldots$, and $\frac{\partial f}{\partial x}(x, n)$ and $\frac{\partial f}{\partial n}(x, n)$ are easily seen to be negative for $x \geqslant 2 \log (3 e / 2)$ and $n \geqslant 16$. Thus, $f(x, n)<0$ for all $x \geqslant 2 \log (3 e / 2)$ and $n \geqslant 16$. As $f(\alpha, n) \geqslant 0$, we have $\alpha<2 \log (3 e / 2)$, as desired. 
Define

$$
\delta=\frac{1-\rho}{e \rho}
$$

Claim 8. We have

$$
(i+1)(1-\rho)^{2} \rho^{i} \leqslant \delta
$$

for all $i \geqslant 0$.

Proof. By the arithmetic mean-geometric mean inequality, we have

$$
\left(\frac{\rho}{i+1}\right)^{i+1}(1-\rho) \leqslant\left(\frac{1}{i+2}\right)^{i+2} \text {. }
$$

Therefore,

$$
(i+1)(1-\rho)^{2} \rho^{i} \leqslant \frac{(1-\rho)}{\rho}\left(\frac{i+1}{i+2}\right)^{i+2} \leqslant \frac{(1-\rho)}{\rho} \frac{1}{e}=\delta,
$$

as desired.

Next we estimate $c_{b}$. Define $\Delta_{b}=z_{b}-z_{b+1}$. Direct calculation shows that

$$
\Delta_{b}=\frac{1}{2}\left((b+1) \rho^{b}-b \rho^{b+1}+(n-b-1) \rho^{n-b}-(n-b) \rho^{n-b-1}\right) .
$$

Claim 9. We have

$$
\Delta_{i+1} \leqslant \Delta_{i} \leqslant \Delta_{i+1}+\delta
$$

for all $1 \leqslant i \leqslant n-1$.

Proof. We have

$$
\begin{aligned}
2\left(\Delta_{i}-\Delta_{i+1}\right)= & (i+1) \rho^{i}-(2 i+2) \rho^{i+1}+(i+1) \rho^{i+2} \\
& +(n-i-1) \rho^{n-i-2}-2(n-i-1) \rho^{n-i-1}+(n-i-1) \rho^{n-i} \\
= & (1-\rho)^{2}\left((i+1) \rho^{i}+(n-i-1) \rho^{n-i-2}\right) .
\end{aligned}
$$

The last term is clearly nonnegative, and it is at most $2 \delta$ by Claim 8 . Thus, the claim holds.

Claim 10. We have

$$
0 \leqslant \Delta_{i} \leqslant \frac{\delta(n-1-2 i)}{2},
$$

for all integers $1 \leqslant i \leqslant(n-1) / 2$.

Proof. As $z_{i}=z_{n-i}$, we have $\Delta_{i}=-\Delta_{n-1-i}$ for all $i$. Thus, if $n$ is odd, we have $\Delta_{(n-1) / 2}=0$. For even $n$, we have $\Delta_{n / 2}=-\Delta_{n / 2-1}$, and $\Delta_{n / 2} \leqslant \Delta_{n / 2-1} \leqslant \Delta_{n / 2}+\delta$ 
by Claim 9 . Thus, $0 \leqslant \Delta_{n / 2-1} \leqslant \delta / 2$. This establishes the claim for $i \in\{(n-1) / 2$, $n / 2-1\}$.

The claim for general $i$ follows directly from Claim 9 by induction on $\lfloor n / 2\rfloor-i$, with the result of the preceding paragraph used as the base case.

Claim 11. We have

$$
c_{b} \leqslant \frac{\delta(n-2 b)}{6}
$$

for every positive integer $2 \leqslant b \leqslant n / 2-1$.

Proof. As in Claim 10, the proof is by induction on $\lfloor n / 2\rfloor-b$, and the base case is $b=\lfloor n / 2\rfloor-1$.

Suppose first that $n$ is even. Then $n=2 b+2$ in the base case, and $c_{b}=2 \Delta_{b} / 3$ by definition. As $0 \leqslant \Delta_{b} \leqslant \delta / 2$ by Claim 10, (13) holds. If $n$ is odd, then $n=2 b+3$, $c_{b}=\Delta_{b} / 2$ and $0 \leqslant \Delta_{b} \leqslant \delta$ by Claim 10, implying that (13) once again holds. This finishes the proof of the base case.

For the induction step, note that

$$
\begin{aligned}
& \frac{(n+1-2 b)(n-1-2 b) c_{b}}{2} \\
& \quad=(n-1-2 b) z_{b}--\sum_{i=b+1}^{n-b-1} z_{i} \\
& \quad=(n-1-2 b) \Delta_{b}+(n-1-2 b) z_{b+1}-\sum_{i=b+1}^{n-b-1} z_{i} \\
& \quad=(n-1-2 b) \Delta_{b}+(n-1-2(b+1)) z_{b+1}-\sum_{i=b+2}^{n-b-2} z_{i} \\
& \quad=(n-1-2 b) \Delta_{b}+\frac{(n-1-2 b)(n-3-2 b) c_{b+1}}{2}
\end{aligned}
$$

Thus,

$$
c_{b}=\frac{2 \Delta_{b}+(n-3-2 b) c_{b+1}}{(n+1-2 b)} .
$$

Using the bounds on $\Delta_{b}$ from Claim 10 and the induction hypothesis applied to $c_{b+1}$, we obtain

$$
0 \leqslant c_{b} \leqslant \frac{\delta(n-1-2 b)+\delta(n-3-2 b)(n-2-2 b) / 6}{n+1-2 b}=\frac{\delta(n-2 b)}{6},
$$

as desired. 
Claim 12. We have

$$
\rho^{b}-\rho^{n-b} \geqslant 4 c_{b}
$$

for all positive integers $2 \leqslant b \leqslant n / 2-1$.

Proof. Let

$$
f(x)=\rho^{x}-\rho^{n-x}-\frac{2 \delta(n-2 x)}{3} .
$$

By Claim 11, it suffices to show that $f(x) \geqslant 0$ for all $x \leqslant n / 2$. As $f(n / 2)=0$, and $f^{\prime \prime}(x) \geqslant 0$ for $x \leqslant n / 2$, it is enough to verify that $f^{\prime}(n / 2) \leqslant 0$, that is,

$$
-2 \rho^{n / 2} \log \rho \geqslant \frac{4}{3} \delta=\frac{4(1-\rho)}{3 e \rho} .
$$

As $-\log \rho \geqslant 1-\rho$, the above is implied by Claim 7 .

The next claim finishes the proof of Theorem 3.

Claim 13. We have $\pi_{x y z} \geqslant 0$ for $(x, y, z) \in T, x, y, z \geqslant 1$.

Proof. Assume that $x \leqslant y \leqslant z$. Suppose that the component corresponding to $(x, y, z)$ is negative in $\boldsymbol{m}(b \rightarrow a)$ for some $2 \leqslant b<a \leqslant n / 2$. Direct examination of (7) shows that, if $z>n / 2$, then

(N1) either $b \in\{2 x, 2 x+1\}$ and $a=x+y$, in which case $(x, y, z)=(\lfloor b / 2\rfloor$, $a-\lfloor b / 2\rfloor, n-a)$, or

(N2) $b \in\{2 x, 2 x-1\}, a=y$, in which case $(x, y, z)=(\lceil b / 2\rceil, a, n-a-\lceil b / 2\rceil)$. If $z<n / 2$, then

(N2) either $b \in\{2 x, 2 x-1\}$ and $a=y,(x, y, z)=(\lceil b / 2\rceil, a, n-a-\lceil b / 2\rceil)$ as before or

(N3) $b \in\{2 x, 2 x-1\}, a=z$, in which case $(x, y, z)=(\lceil b / 2\rceil, n-a-\lceil b / 2\rceil, a)$. If $z=n / 2$, then any of the above cases can potentially occur, but in cases (N1) and (N3), the component of $\boldsymbol{m}(b \rightarrow a)$ corresponding to $(x, y, z)$ is equal to $c_{b} / 2$ rather than $c_{b}$.

Suppose first that $x<y<z$. By the above analysis, the total negative contribution to $\pi_{x y z}$ of vectors $\boldsymbol{m}(b \rightarrow a)$ for $2 \leqslant b<a \leqslant n / 2$ is at most $4 \max \left\{c_{2 x}, c_{2 x+1}, c_{2 x-1}\right\} \leqslant \rho^{x}-\rho^{n-x}$, where the last inequality holds by Claim 12 . Thus,

$$
\pi_{x y z} \geqslant \beta_{x y z}-\rho^{x}-\rho^{n-x} \geqslant\left(\rho^{z}-\rho^{n-y}\right)+\left(\rho^{y}-\rho^{n-z}\right)>0,
$$

as desired. 
Finally, suppose that $x \leqslant y \leqslant z$, and one of these inequalities is nonstrict. Then a vector $\boldsymbol{m}(b \rightarrow a)$ can only contribute negatively to $\pi_{x y z}$ if $x<y=z$ and $(x, y$, $z)=(\lceil b / 2\rceil, a, n-a-\lceil b / 2\rceil)$. Note that in this case, the component of $\boldsymbol{m}(b \rightarrow a)$ corresponding to $(x, y, z)$ is equal to $2 c_{b}$, rather than $c_{b}$, but the bound $4 \max \left\{c_{2 x}\right.$, $\left.c_{2 x+1}, c_{2 x-1}\right\}$ on the total negative contribution established in the previous case still holds.

\section{Acknowledgements}

We would like to thank the anonymous referee and Lisa Sauermann for pointing out an error in the estimates used in the proof of Theorem 3 in an earlier version of this paper. The author is supported by an NSERC Discovery grant.

\section{Conflict of Interest: None}

\section{References}

[1] J. Blasiak, T. Church, H. Cohn, J. A. Grochow, E. Naslund, W. F. Sawin and C. Umans, 'On cap sets and the group-theoretic approach to matrix multiplication', Discrete Anal. (2017), Paper No. 3, 27 pp.

[2] E. Croot, V. F. Lev and P. P. Pach, 'Progression-free sets in $\mathbb{Z}_{4}^{n}$ are exponentially small', Ann. of Math. (2) 185(1) (2017), 331-337.

[3] J. S. Ellenberg and D. Gijswijt, 'On large subsets of $\mathbb{F}_{q}^{n}$ with no three-term arithmetic progression', Ann. of Math. (2) 185(1) (2017), 339-343.

[4] J. Fox and L. M. Lovász, 'A tight bound for Green's arithmetic triangle removal lemma in vector spaces', Adv. Math. 321 (2017), 287-297.

[5] R. Kleinberg, W. Sawin and D. E. Speyer, 'The growth rate of tri-colored sum-free sets', Discrete Anal. (2018), Paper No. 12, arXiv:1607.00047v1, 10 pp.

[6] L. Pebody, 'Proof of a conjecture of Kleinberg-Sawin-Speyer', Discrete Anal. (2018), Paper No. 13, 7 pp. 